\title{
Angelica sinensis extract inhibits RANKL-mediated osteoclastogenesis by down-regulated the expression of NFATc1 in mouse bone marrow cells
}

\author{
Lingbo Kong ${ }^{1 \dagger}$, Qinpeng Zhao ${ }^{1 \dagger}$, Xiaodong Wang ${ }^{1}$, Jinyu Zhu ${ }^{2}$, Dingjun Hao ${ }^{1^{*}}$ and Chongfei Yang ${ }^{2^{*}}$
}

\begin{abstract}
Background: Destructive erosion of bone or osteolysis is a major complication of inflammatory conditions such as rheumatoid arthritis (RA), periodontal disease, and periprosthetic osteolysis. Natural plant-derived products have received recent attention as potential therapeutic and preventative drugs in human disease.

Methods: The effect of Angelica sinensis (AS) extract on RANKL-induced osteoclast differentiation was examined in this study. The osteoclast precursor cell line bone marrow macrophages (BMMs) was cultured and stimulated with RANKL followed by treatment with AS at several doses. Gene expression profiles of c-Fos, c-Jun, NFATc1, TRAP, and OSCAR were sequentially evaluated.

Results: AS extract inhibited RANKL-mediated osteoclast differentiation in BMMs in a dose-dependent manner without any evidence of cytotoxicity. AS extract strongly inhibited p38, ERK, JNK, p65 phosphorylation and I-KB degradation in RANKL-stimulated BMMs. AS extract also inhibited the mRNA expression of c-Fos, c-Jun, NFATc1, TRAP, and OSCAR in RANKL-treated BMMs. Moreover, RANKL-induced c-Fos, c-Jun and NFATc1 protein expression was suppressed by AS extract.

Conclusions: These results collectively suggested that AS extract demonstrated inhibitory effects on RANKL-mediated osteoclast differentiation in bone marrow macrophages in vitro, indicating that AS may therefore serve as a useful drug in the prevention of bone loss.
\end{abstract}

Keywords: Angelica sinensis, Osteoclastogenesis, NFATc1, BMMs

\section{Background}

Osteoclasts are derived from hematopoietic stem cells, which are unique multinucleated cells responsible for bone resorption [1]. Osteoblasts and stromal cells express receptor activators of the nuclear factor-kB (NF-kB) ligand (RANKL) and macrophage colony-stimulating factor (MCSF) [2]. M-CSF induces expression of RANKL receptor (RANK) as well as supports survival and proliferation in early stage precursors of osteoclast lineages in mouse bone marrow cells [3]. RANKL is a member of the tumor necrosis factor (TNF) family and binds to the RANK receptor expressed in osteoclast precursor cells [4]. The binding

\footnotetext{
*Correspondence: dhao.honghui@outlook.com; jinyuzhu@outlook.com ${ }^{\dagger}$ Equal contributors

${ }^{1}$ Hong-Hui Hospital, Xi'an Jiaotong University College of Medicine, 710054 Xi'an, China

${ }^{2}$ Institute of Orthopedic Surgery, Xijing Hospital, Fourth Military Medical University, 710054 Xi'an, China
}

of RANKL and RANK on osteoclast progenitor cells triggers the activation of tumor necrosis factor receptorassociated factor 6 (TRAF6) [5] and subsequently the activation of NF-kB and mitogen-activated protein kinases (MAPKs), such as extracellular signal-regulated kinase $1 / 2$ (ERK1/2), p38 and stressactivated protein kinase/c-Jun Nterminal kinase (SAPK/JNK) [6,7]. Although more detailed mechanism is still expected to be unveiled, the major signaling events triggered upon RANK ligation include recruitment of TRAF6, the activation of transcription factors NF- $\mathrm{kB}$, c-Fos, AP-1, and nuclear factor of activated T cells (NFATc1) [2,5,8-11]. NFATc1 is a downstream transcription factor in the RANKL/RANK signal pathway and as a key molecule of osteoclastogenesis, NFATc1 induces a series of osteoclast-specific genes, including tartrate-resistant acid phosphatase (TRAP), osteoclastassociated receptor (OSCAR) and cathepsin $\mathrm{K}-[12,13]$. 
c-Fos is also an essential transcription factor for osteoclastogenesis and positively regulates osteoclastogenesis via NFATc1 activation [14].

Many plant-derived natural products have been used in traditional medicine for the treatment of various diseases. Several compounds derived from natural products have been recently reported to possess inhibitory effects on osteoclast differentiation and function, leading to decreased bone loss in vivo [1]. Angelica sinensis (AS) has been used to regulate menstruation, an inflammatory syndrome, in Asia for thousands of years. Recently, a component of AS extract, ligustilide has been reported to regulate several extracellular signaling pathways, including ERK1/2, p38 and SAPK/JNK [15]. Additionally, our previous study demonstrated that AS extract could inhibit wear debris particles-induced bone resorption by attenuating proinflammatory cytokines [16]. However, the effects of AS extract on RANKL and M-CSF functions vital to osteoclast differentiation are not clarified. In this study, we aimed to investigate the effects of AS extract on signaling pathways involved in osteoclast differentiation, activation, and survival in vitro.

\section{Methods}

\section{Herb preparation}

Dry root slices of a popular Chinese herb, Angelica sinensis (Dang Gui), was obtained from Tong Ren Tang (Tong Ren Tang Group Co., Ltd.; Beijing, China) and extracted in water $\left(85^{\circ} \mathrm{C}\right)$ for $4 \mathrm{~h}$. The water-soluble fraction was cleared sequentially by centrifugation $(3300 \times \mathrm{g}$, $20 \mathrm{~min}, 4^{\circ} \mathrm{C}$ ) and filtration through a $0.2 \mathrm{~mm}$ filter [17]. From $10 \mathrm{~g}$ dry Angelica sinensis root, about $0.8 \mathrm{~g}$ yellow, powdery substance was recovered after lyophilization.

\section{Reagents and antibodies}

Human RANKL and M-CSF was obtained from Peprotech (London, UK). The XTT assay kit was obtained from Roche (Indianapolis, IN, USA). Antibodies for cFos, c-Jun and nuclear factor of activated $\mathrm{T}$ cells 1 (NFATc1) were purchased from Santa Cruz Biotechnology (Santa Cruz, CA, USA), and Western blot antibodies for phosphor-p65, p-65, phosphor-ERK, ERK, phosphorJNK, JNK, phosphor-p38, p38, and I-kB were from Santa Cruz Biotechnology Inc. (Santa Cruz, CA, USA); $\beta$-actin antibody was purchased from Sigma-Aldrich, Inc. (St. Louis, MO, USA).

\section{Osteoclast differentiation}

All the animal work and approach have been approved by the IACUC of the Hong-Hui Hospital, Xi'an Jiaotong University College of Medicine and conducted strictly followed by "the institutional guidelines for the care and use of laboratory animals at the Jiaotong University College of Medicine". Bone marrow cells were obtained by flushing the femurs and tibiae of 5-week-old ICR mice with $\alpha$-minimum essential medium ( $\alpha$-MEM; Gibco BRL, Gaithersburg, MD, USA) and suspended in $\alpha$ MEM supplemented with 10\% fetal bovine serum (FBS; Gibco BRL, Gaithersburg, MD, USA). Non-adherent cells were collected and cultured for 3 days in the presence of M-CSF $(20 \mathrm{ng} / \mathrm{ml})$. Floating cells were discarded and adherent cells on dish bottoms were classified as bone marrow derived macrophages (BMMs). BMMs were seeded at $3.5 \times 10^{4}$ cells/well in $\alpha$-MEM/10\% FBS, and were cultured in the presence of M-CSF $(20 \mathrm{ng} / \mathrm{ml})$ and RANKL ( $40 \mathrm{ng} / \mathrm{ml}$ ) for 4 days in the presence or absence of AS extract. Osteoclasts were identified by staining for tartrate-resistant acid phosphatase (TRAP) activity, as described below. TRAP-positive multinucleated cells with greater than three nuclei were counted as osteoclasts. Cytotoxicity assay for AS extract treated BMMs were plated in 96-well plates at a density of $1 \times 10^{4}$ cells/well in triplicate. Cells were treated with M-CSF $(20 \mathrm{ng} / \mathrm{ml})$ and increasing concentrations of AS extract were added to the mix. Cells were incubated for 3 days. After 3 days, XTT reagent $(50 \mu \mathrm{l})$ was added to each well. Wells were incubated for $4 \mathrm{~h}$. The optical density at $450 \mathrm{~nm}$ was analyzed with an ELISA reader.

\section{Clonogenic assay}

RAW 264.7 cells were seeded in 48-well plates at a density of $3 \times 10^{3}$ cells/well in triplicate and cultured for 4 days in the presence of increasing concentrations of AS extract. After 4 days, the cells were fixed and stained with Hematoxylin Sigma-Aldrich, Inc. (St. Louis, MO, USA). Colonies with 50 or greater cells were counted.

Real time RT-PCR analysis for c-Fos, c-Jun, NFATc1, TRAP, osteoclast-associated receptor (OSCAR)

Total RNA was isolated with TRIzol reagent (Invitrogen Inc., USA) per the manufacturer's instructions. RNA $(1 \mu \mathrm{g})$ was reverse transcribed using oligo dT primers $(10 \mu \mathrm{g})$ and dNTPs $(10 \mathrm{mM})$. The mixture was incubated at $65^{\circ} \mathrm{C}$ for $5 \mathrm{~min}$, and cDNA was produced by incubating at $42^{\circ} \mathrm{C}$ for $50 \mathrm{~min}$ with first strand buffer $(50 \mathrm{mM}$ Tris$\mathrm{HCl}, \mathrm{pH}$ 8.3, $75 \mathrm{mM} \mathrm{KCl}, 3 \mathrm{mM} \mathrm{MgCl}_{2}$ ), $100 \mathrm{mM}$ DTT, RNase inhibitor, and Superscript II reverse transcriptase (Invitrogen). The cDNA was amplified using the following primer sets: c-Fos, 5' -CTGGTGCAGCCCACTCTGGTC3' (forward) and 5'-CTTTCAGCAGATTGGCAATCTC3' (reverse); c-Jun, 5'-ACT CGG ACC TTC TCA CGT CG- 3' (forward) and 5'-TAG ACC GGA GGC TCA CTG TG -3' (reverse); NFATc1, 5' -CTCGAAAGACAG CACTGGAGCAT-3' (forward) and 5'-CGGCTGCCTTC CGTCTCATAG-3' (reverse); TRAP, 5' -CTGGAGTGCA CGATGCCAGCGACA-3' (forward) and 5'-TCCGTGC TCGGCGATGGACCAGA-3' (reverse); OSCAR, 5'-CTG CTGGTAACGGATCAGCTCCCCAGA-3' (forward) and 
5'-CCAAGGAGCCAGAACCTTCGAAACT-3' (reverse); and GAPDH, 5' -ACCACAGTCCATGCCATCAC-3' (forward) and 5'-TCCACCACCCTGTTGCTGTA- 3'(reverse). PCR was performed using the QuantiTect SYBR Green PCR kit (Qiagen) in triplicates according to the manufacturer's instructions. Relative levels of c-Fos, c-Jun, NFATc1, TRAP, and OSCAR were normalized to GAPDH.

\section{Western blot analysis}

BMMs or osteoclasts were lysed in a buffer containing $50 \mathrm{mM}$ Tris- $\mathrm{HCl}, 150 \mathrm{mM} \mathrm{NaCl}, 5 \mathrm{mM}$ EDTA, 1\% Triton X-100, $1 \mathrm{mM}$ sodium fluoride, $1 \mathrm{mM}$ sodium vanadate, $1 \%$ deoxycholate, and protease inhibitors. The lysates were centrifuged at $14,000 \times \mathrm{g}$ for $20 \mathrm{~min}$ and supernatants were collected. Protein concentrations of supernatants were determined. Cellular proteins $(30 \mu \mathrm{g})$ were resolved by $8-10 \%$ sodium dodecyl sulfatepolyacrylamide gel electrophoresis (SDS-PAGE) and were transferred to polyvinylidene difluoride membranes (Milipore, Bedford, MA, USA). Non-specific interactions were blocked with $5 \%$ skim milk for $2 \mathrm{~h}$ and were then probed with the appropriate primary antibodies. Membranes were incubated with the appropriate secondary antibodies attached to horseradish peroxidase, and immunoreactivity was detected with enhanced chemiluminescence reagents. Densitometric values were quantified for each band with the Image Pro-plus program version 4.0.

\section{Statistical analysis}

All data are expressed as means \pm standard deviation (SD). Statistical analysis was done using SPSS software package ver. 11.0 (SPSS, Chicago, IL); one-way ANOVA was used for comparison among the different groups. Post hoc testing of differences between groups was performed by using Duncan's test when the ANOVA was
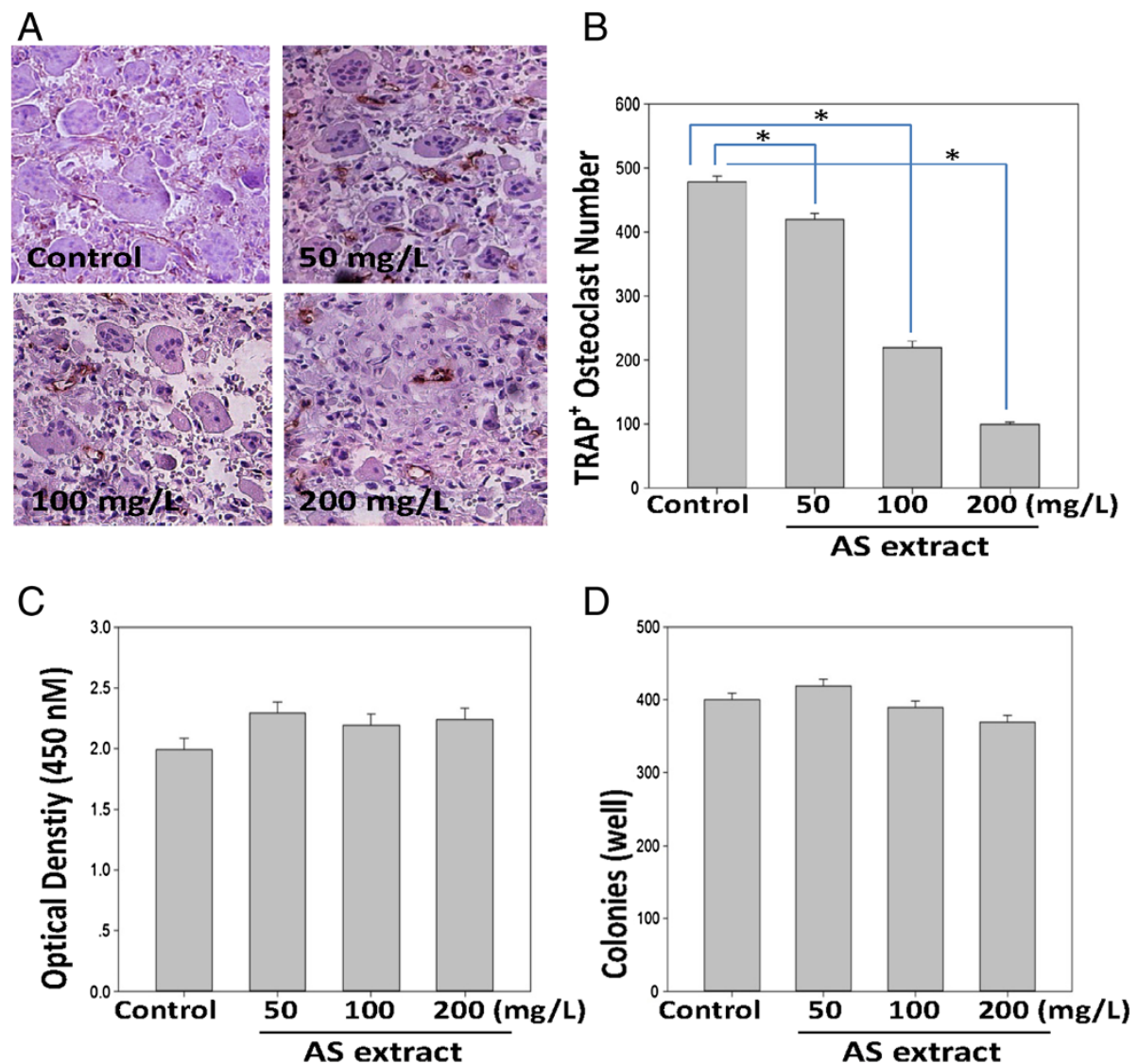

Figure 1 Effect of AS extract on RANKL-induced osteoclast differentiation. (A) BMMs were cultured for 4 days with M-CSF (20 ng/ml) and RANKL $(40 \mathrm{ng} / \mathrm{ml})$ in the presence of varying concentrations of AS extract. Cells were fixed with $3.7 \%$ formalin, permeabilized with $0.1 \%$ Triton X-100, and stained with TRAP solution. (B) TRAP-positive cells were counted as osteoclasts. Asterisk indicates a statistically significant difference $(p<0.05)$ between control and treated. (C) Cytotoxicity of AS extract on BMMs. BMMs were cultured for 3 days with varying concentrations of AS extract in the presence of M-CSF $(20 \mathrm{ng} / \mathrm{ml})$. XTT solution $(50 \mu \mathrm{l})$ was added to each well after 3 days, and plates were incubated for $4 \mathrm{~h}$. The optical density was measured at $450 \mathrm{~nm}$. (D) RAW 264.7 cells were seeded at 3000 cells/plate in 48-well plates and cultured with the indicated concentrations of AS extract for 4 days. After 4 days, the cells were fixed and stained with Hematoxylin. Colonies with 50 or greater cells were counted. Similar results were obtained in at least 3 independent experiments. 
significant. All results were considered to be significant at the $5 \%$ critical level $(P<0.05)$.

\section{Results}

Inhibition of osteoclast differentiation by AS extract Osteoclasts were generated from mouse BMMs in the presence of M-CSF $(20 \mathrm{ng} / \mathrm{ml})$ plus RANKL (40 ng/ml) to verify the effects of AS extract in osteoclastogenesis. The BMMs of the control group differentiated into mature TRAP-positive multinucleated osteoclasts while AS extract reduced the formation and numbers of TRAPpositive multinucleated cells in a dose-dependent manner (Figure 1A, B).

\section{The cytotoxic effect of AS extract}

AS extract generated a highly negative effect on osteoclastogenesis. We measured the effects of AS extract on bone marrow cells with the XTT assay to exclude the possibility that the inhibition was due to cytotoxicity. AS extract demonstrated no cytotoxic effects at the same doses which effectively inhibited osteoclast formation (Figure 1C). Also, AS extract did not affect in RAW 264.7 cells colony formation (Figure 1D), suggesting that osteoclastogenesis suppression by AS extract was not due to toxic effects on BMMs.

\section{AS extract inhibits a variety of signals transduced by RANKL}

RANKL activates a variety of signal transducers that are involved in osteoclastogenesis, including p38, JNK, ERK, transcription factor NF- $\mathrm{kB}$ and I- $\mathrm{kB}$, which are recognized as the key factors of osteoclast differentiation $[2,3]$. Osteoclast precursors were pretreated with AS extract and stimulated with RANKL at various time points. Different signaling pathways were observed. Activation of ERK,

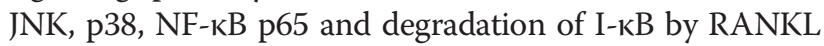
were all significantly inhibited by AS extract (Figure 2).

\section{RANKL induced c-Fos, c-Jun, NFATc1, TRAP, and OSCAR mRNA expression is reduced by AS extract}

Osteoclast differentiation is regulated by the induction of various genes in response to RANKL and RANK binding. The c-Fos, c-Jun and NFATc1 genes have an essential role in the osteoclast differentiation $[2,13]$, and NFATc1 regulates OSCAR expression [18]. We examined the effects of AS extract on the RANKL-induced regulation of c-Fos, c-Jun and NFATc1 expression, and to assess whether there were any effects on TRAP and OSCAR expression. Osteoclast precursors were pretreated with AS extract and further stimulated with RANKL at various time points. Results revealed that cFos, c-Jun and NFATc1 mRNA levels were increased in response to RANKL, but c-Fos, c-Jun and NFATc1 mRNA expression was significantly inhibited by AS extract.

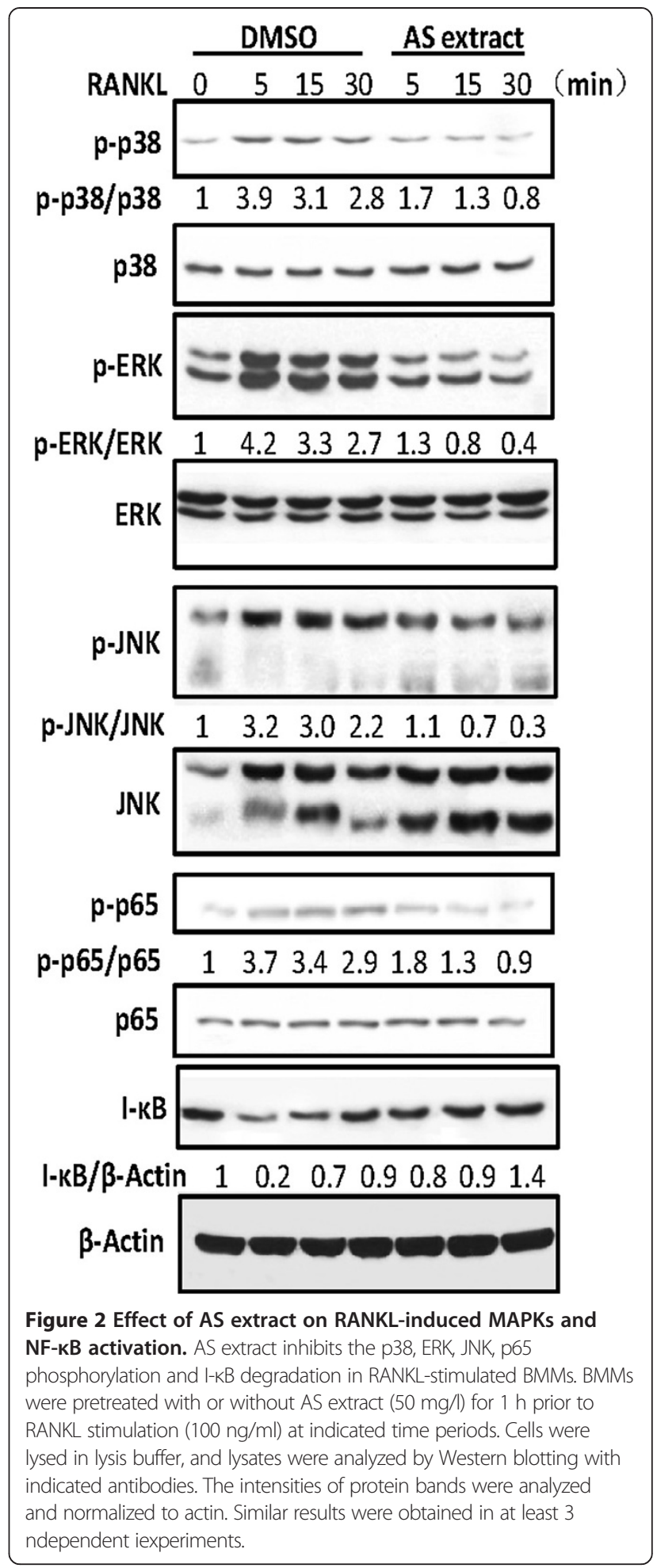

Moreover TRAP and OSCAR mRNA expression was also significantly inhibited by AS extract (Figure 3A). This raises the possibility that, AS extract may inhibit osteoclast differentiation through the inhibition of RANKL-induced c-Fos, c-Jun and NFATc1 expression. 


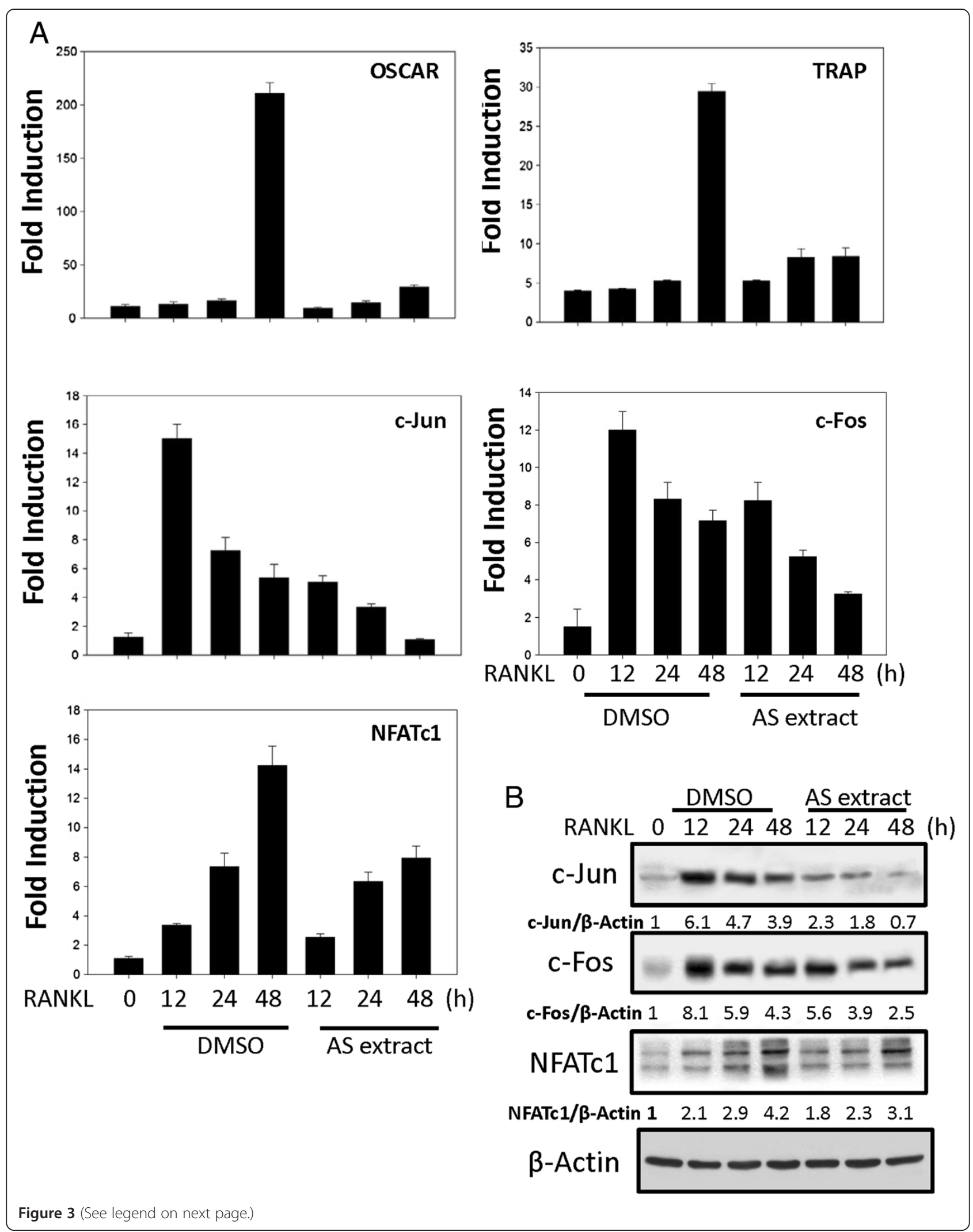


(See figure on previous page.)

Figure 3 AS extract suppresses the mRNA expression of c-Fos, c-Jun, NFATc1, OSCAR and TRAP in BMMs treated with RANKL. (A) BMMs were pretreated with or without AS extract $(50 \mathrm{mg} / \mathrm{l})$ for $1 \mathrm{~h}$ and with RANKL $(100 \mathrm{ng} / \mathrm{ml})$ for the indicated time. Total RNA was isolated using TRIzol, and $1 \mu \mathrm{g}$ of total RNA was used to transcribe CDNA. CDNA $(1 \mu \mathrm{l})$ was used as a template for real time RT-PCR. The mRNA expression of the indicated genes was analyzed by real time RT-PCR. (B) AS extract inhibits the expression of c-Jun, c-Fos and NFATc1 induced by RANKL. BMMs were pretreated with or without AS extract $(50 \mathrm{mg} / \mathrm{l})$ for $1 \mathrm{~h}$ and were treated with RANKL $(100 \mathrm{ng} / \mathrm{ml})$ for the indicated time. Cells were lysed in the lysis buffer, and lysates were analyzed by Western blotting with antibodies against c-Jun, c-Fos, NFATc1 and actin. The intensities of the protein bands were analyzed and normalized to actin. Similar results were obtained in at least 3 independent experiments.

\section{AS extract also inhibits c-Fos, c-Jun and NFATc1 protein expression}

Western blots were examined to verify the effects of AS extract on c-Fos, c-Jun and NFATc1 protein expression. c-Fos, c-Jun and NFATc1 protein levels were increased in response to RANKL, but c-Fos, c-Jun and NFATc1 expression was significantly inhibited by AS extract (Figure 3B). These results demonstrated that the inhibitory effects of AS extract involved the inhibition of major transcription factors such as c-Fos, c-Jun and NFATc1.

\section{Discussion}

Bone resorption by osteoclasts is frequently caused by excessive RANKL signaling which has been a valuable target for the treatment of pathological bone loss. RANKL activates a series of major intracellular signal transducing pathways including NFATc1, NF- $\mathrm{k}$, phosphoinositide 3 kinase (PI3K)-Akt, JNK, ERK, and p38 MAPK. AS is one of the most popular Chinese herbs in both clinical field and research field. Su et al. [15] reported that a component of AS extract: ligustilide suppresses the LPS-induced production of NO, PGE2 and TNF- $\alpha$ by inhibiting both IKK/NF-kB and MAPK/AP-1 signaling. Recently we [16] demonstrated that AS extract could inhibit bone resorption by attenuating proinflammatory cytokines. However, the effect of AS extract on RANKL-induced osteoclast formation, especially RANKLmediated intracellular signal pathway still is an interesting question that remains to be investigated.

The MAPK group of enzymes selectively phosphorylates serine and threonine residues in response to extracellular stimuli and transmits the stimuli from the cell surface to the nucleus [7]. MAPK is primarily composed of JNK, ERK, and p38 in mammalian cells. RANKL and RANK receptor binding expressed in osteoclast precursors provides a link between distinct signaling molecules such as JNK, ERK, and p38 MAPK [1]. p38 MAPK signaling is particularly crucial in the early stages of osteoclast differentiation as it promotes the activity of microphthalmia-associated transcription factor (MITF) and TRAP expression. Inhibition of p38 MAPK with SB203580 has a negative effect on osteoclast formation [19]. AS extract suppressed RANKL-induced the phosphorylation of p38, ERK and JNK activity in the present study (Figure 2).
The activation of signaling molecules induces transcription factors such as NF-kB, NFATc1, and AP-1 that are essential for osteoclast differentiation [20]. NF- $k B$ is an important signal mediator for inflammatory and immune reactions and is a major transcription factor for RANKL-activated osteoclastogenesis [21]. I- $\kappa B$ is attached to NF- $\mathrm{kB}$ preventing it from migrating into the nucleus, and phosphorylation with $\mathrm{I}-\mathrm{kB}$ kinase (IKK) separates the two proteins. Subsequent ubiquitination and proteosome degradation of $\mathrm{I}-\mathrm{kB}$ allows the transfer of NF- $\mathrm{kB}$ into the nucleus and transcription of the target gene [22]. Although our previous study showed that one factor of AS extract: ligustilide decreases NF- $\mathrm{kB}$ luciferase activity in a reporter assay [16], we also confirmed that AS extract inhibits $\mathrm{NF}-\mathrm{kB} / \mathrm{I}-\mathrm{kB}$ protein expression in this experiments. These results are consistent with RANKL activation of NF- $\mathrm{kB}$ in osteoclastic precursor cells through IKK activation and susequent I- $\mathrm{K} B$ phosphorylation and degradation (Figure 2).

NFATc1 is a master regulator of osteoclastogenesis which autoamplifies and conducts the expression of osteoclast specific genes such as activator protein-1 (AP-1), TRAP, calcitonin receptor, OSCAR, and cathepsin K [10,23-25]. The RANKL-induced NFATc1 expression is mediated by the activation of AP-1 consisting of c-Fos and c-Jun [26,27]. c-Fos was reported to be critical for transcriptional activation of NFATc1 in RANKL-induced osteoclastogenesis [7]. Putative c-Fos binding sites were mapped in the promoter region of NFATc1, and the NFATc1 expression was abolished in the osteoclast precursors lacking c-Fos [11].On the other hand, Ikeda et al. [26] reported that RANKL could not induce expression of NFATc1 in dominant-negative c-Jun transgenic mouse. It is possible that the induction of NFATc1 may be cooperatively upregulated by c-Fos and c-Jun, which make up each other for the induction [28]. The present study demonstrates the reduced both c-Fos and c-Jun expression in AS extract treated BMM cells (Figure 3). Therefore, the reduced c-Fos and c-Jun expression is suggested to cause the impaired activation of NFATc1, followed by the inhibition of RANKLinduced osteoclast formation. Additionally mRNA levels of major osteoclast marker TRAP and OSCAR was also inhibited by AS extract (Figure 3). These data suggest that the AP-1 transcription factor is the targets of AS extractinduced inhibition of osteoclastogenesis. 


\section{Conclusions}

In summary, the present study demonstrated that AS inhibited osteoclastogenesis from macrophages and bone resorption in vitro. AS also reduced the RANKL-induced expression of osteoclastic marker genes. In addition, AS attenuated RANKL-induced ERK, p38, JNK, NF-kB, AP-1 and NFATc1 activation. Although additional experiments are needed to confirm the efficacy of AS in treating disease conditions in vivo, our results indicate that AS has potential as a therapy for disorders associated with bone loss.

\section{Competing interests}

The authors declare that they have no competing interests.

\section{Authors' contributions}

$\mathrm{DH}, \mathrm{CY}, \mathrm{LK}$ : conception and design, analysis and interpretation of data; draft the manuscript and revise it critically for important intellectual content; final approval of the version to be published: acquisition of data, analysis and interpretation of data; QZ: acquisition of data, analysis and interpretation of data; DW: acquisition of data, draft the manuscript. JZ, DH: conception and design, revise the manuscript critically for important intellectual content, final approval of the version to be published, account for all aspects of the work in ensuring that questions related to the accuracy or integrity of any part of the work are appropriately investigated and resolved. All authors read and approved the final manuscript. All authors read and approved the final manuscript.

\section{Acknowledgements}

The authors wish to thank Guo Hua (Honghui hospital) for overseeing the conduct of the study, Rui Ma, the Fourth Military Medical University (Xi'an, China) for statistical analysis assistance and Shu Zhu for overseeing data management. We thank Lifeng Yu for regulatory and quality activities and for his technical support. We thank Xiaorui Cao, PhD, for his assistance with manuscript preparation and review.

Received: 14 May 2014 Accepted: 10 December 2014

Published: 12 December 2014

\section{References}

1. Han Bok K, Byeong Ki L: Inhibition of osteoclast differentiation and bone resorption by rotenone, through down-regulation of RANKL-induced c-Fos and NFATc1 expression. Bone 2010, 46:724-731.

2. Boyle WJ, Simonet WS, Lancey DL: Osteoclast differentiation and activation. Nature 2003, 423:337-342.

3. Arai F, Miyamoto T, Ohneda O, Inada T, Sudo T, Brasel K: Commitment and differentiation of osteoclast precursor cells by the sequential expression of c-Fms and receptor activator of nuclear factor kappaB (RANK) receptors. J Exp Med 1999, 190:1741-1754.

4. Suda T, Takahashi N, Udagawa N, Jimi E, Gillespie MT, Martin TJ: Modulation of osteoclast differentiation and function by the new members of the tumor necrosis factor receptor and ligand families. Endocr Rev 1999, 20:345-357.

5. Kobayashi N, Kadono Y, Naito A, Matsumoto K, Yamamoto T, Tanaka S: Segregation of TRAF6-mediated signaling pathways clarifies its role in osteoclastogenesis. EMBO J 2001, 20:1271-1280.

6. Hotokezaka H, Sakai E, Kanaoka K, Saito K, Matsuo K, Kitaura H: U0126 and PD98059, specific inhibitors of MEK, accelerate differentiation of RAW264.7 cells into osteoclast-like cells. J Biol Chem 2002. 277:47366-47372.

7. Matsuo K, Galson DL, Zhao C, Peng L, Laplace C, Wang KZ: Nuclear factor of activated T-cells (NFAT) rescues osteoclastogenesis in precursors lacking c-Fos. J Biol Chem 2004, 279:26475-26480.

8. David JP, Sabapathy K, Hoffmann O, Idarraga MH, Wagner EF: JNK1 modulates osteoclastogenesis through both c-Jun phosphorylation-dependent and independent mechanisms. J Cell Sci 2002, 115:4317-4325.

9. Lee SE, Woo KM, Kim SY, Kim HM, Kwack K, Lee ZH: The phosphatidylinositol 3-kinase, p38, and extracellular signal-regulated kinase pathways are involved in osteoclast differentiation. Bone 2002, 30:71-77.

10. Takayanagi H, Kim S, Koga T, Nishina H, Isshiki M, Yoshida H: Induction and activation of the transcription factor NFATc1 (NFAT2) integrate RANKL signaling in terminal differentiation of osteoclasts. Dev Cell 2002, 3:889-901.

11. Matsuo K, Owens JM, Tonko M, Elliott C, Chambers TJ, Wagner EF: Fos is a transcriptional target of c-Fos during osteoclast differentiation. Nat Genet 2000, 24:184-187.

12. Nakashima T, Takayanagi H: Osteoimmunology: cross talk between the immune and bone systems. J Clin Immunol 2009, 29:555-567.

13. Zhao Q, Wang X, Liu Y, He A, Jia R: NFATc1: function in osteoclasts. Int J Biochem Cell Biol 2010, 42:576-579.

14. Grigoriadis AE, Wang ZQ, Cecchini MG, Hofstetter W, Felix R, Fleisch HA: c-Fos: a key regulator of osteoclast-macrophage lineage determination and bone remodeling. Science 1994, 266:443-448.

15. Su YW, Chiou WF, Chao SH: Ligustilide prevents LPS-induced iNOS expression in RAW 264.7 macrophages by preventing ROS production and down-regulating the MAPK, NF-KB and AP-1signaling pathways. Int Immuno Pharmacol 2011, 11:1166-1172.

16. Yang C, Niu S, Lifeng Y: The aqueous extract of Angelica sinensis, a popular Chinese herb, inhibits wear debris-Induced Inflammatory osteolysis in mice. J Surg Res 2012, 176:476-483.

17. Wang H, Li W, Li J: The aqueous extract of a popular herbal nutrient supplement, Angelica sinensis, protects mice against lethal endotoxemia and sepsis. J Nutr 2006, 136:360.

18. Kim K, Kim JH, Lee J, Jin HM, Lee SH, Fisher DE: Nuclear factor of activated $T$ cells $\mathrm{c} 1$ induces osteoclast-associated receptor gene expression during tumor necrosis factor-related activation-induced cytokine mediated osteoclastogenesis. J Biol Chem 2005, 280:35209-35216.

19. Matsumoto M, Sudo T, Saito T, Osada H, Tsujimoto M: Involvement of p38 mitogen-activated protein kinase signaling pathway in osteoclastogenesis mediated by receptor activator of NF-kappa B ligand (RANKL). J Biol Chem 2000, 275:31155-31161.

20. Takayanagi H: Osteoimmunology. Nat Rev Immunol 2007, 7:292-304

21. lotsova V, Caamano J, Loy J, Yang Y, Lewin A, Bravo R: Osteopetrosis in mice lacking NF-kappaB1 and NF-kappaB2. Nat Med 1997, 3:1285-1289.

22. Hayden MS, Ghosh S: Signaling to NF-KB. Genes Dev 2004, 18:2124-2195.

23. Crotti TN, Flannery M, Walsh NC, Fleming JD, Goldring SR, McHugh KP: NFATc1 regulation of the human beta 3 integrin promoter in osteoclast differentiation. Gene 2006, 372:92-102.

24. Kim Y, Sato K, Asagiri M, Morita I, Soma K, Takayanagi H: Contribution of nuclear factor of activated $T$ cells $\mathrm{c} 1$ to the transcriptional control of immunoreceptor osteoclast-associated receptor but not triggering receptor expressed by myeloid cells-2 during osteoclastogenesis. J Biol Chem 2005, 280:32905-32913.

25. Matsumoto M, Koqawa M, Wada S, Takayangi H, Tsujimoto M, Katayama S: Essential role of p38 mitogen-activated protein kinase in cathepsin $\mathrm{K}$ gene expression during osteoclastogenesis through association of NFATC1 and PU.1. J Biol Chem 2004, 279:45969-45979.

26. Ikeda F, Nishimura R, Matsubara T, Tanaka S, Inoue J, Reddy SV: Criticalroles of c-Jun signaling in regulation of NFAT family and RANKL-regulated osteoclast differentiation. J Clin Invest 2004, 114:475-484.

27. Wagner EF, Eferl R: Fos/AP-1 proteins in bone and the immune system. Immunol Rev 2005, 208:126-140.

28. Mohamed S: Interleukin-10 inhibits RANKL-mediated expression of NFATc1 in part via suppression of c-Fos and c-Jun in RAW264.7 cells and mouse bone marrow cells. Bone 2007, 41:592-602.

doi:10.1186/1472-6882-14-481

Cite this article as: Kong et al:: Angelica sinensis extract inhibits RANKLmediated osteoclastogenesis by down-regulated the expression of NFATc1 in mouse bone marrow cells. BMC Complementary and Alternative Medicine 2014 14:481. 